\title{
ATUAÇÃO DA FISIOTERAPIA NA REABILITAÇÃO CARDIACA DURANTE AS FASES I E II Uma Revisão da Literatura
}

\author{
Mauro Henrique Moraes Vargas ${ }^{1}$ \\ Régis Vieira² \\ Renato Carvalho Balbueno ${ }^{3}$
}

\begin{abstract}
RESUMO
A reabilitação cardíaca é definida como um conjunto de atividades necessárias para garantir aos pacientes portadores de doenças cardiovasculares as melhores condiç̃oes sociais, mentais e físicas, no intuito de recuperar sua qualidade de vida. 0 objetivo deste estudo é identificar os principais desfechos clínicos resultantes de programas de reabilitação cardíaca nas fase 1 e 2 nos últimos cinco anos, caracterizados por uma revisão da literatura realizada em periódicos localizados nos bancos de dados Lilacs, Scielo e Bireme, publicados nos últimos cinco anos. Foram selecionados oito artigos envolvendo programas de reabilitação cardíaca fases 1 e 2 e, entre os benefícios encontrados destacam-se melhora no escore para dispneia, sensação de esforço, volume corrente, capacidade vital, dor, força dos músculos responsáveis pela respiração, frequência cardíaca, pressão arterial sistólica, capacidade funcional, perfil bioquímico e consequentemente melhora na qualidade de vida dos indivíduos. Pode-se concluir que os protocolos de reabilitação cardíaca envolvendo a associação da fisioterapia convencional com incentivadores e outras técnicas terapêuticas como Epap, Bipap e BS, proporcionam um resultado satisfatório e imediato aos indivíduos que necessitam.
\end{abstract}

Palavras-chave: Reabilitação cardíaca. Fisioterapia. Exercício físico.

\section{PERFORMANCE OF PHYSICAL THERAPY IN HEART REHABILITATION DURING PHASE I AND II - A LITERATURE REVIEW}

\section{ABSTRACT}

Cardiac rehabilitation it is defined as activities needed to allow patients with cardiovascular disease have a best social, mental and physical condition in order to improve their quality of life. The aim of this study is to identify the major clinical outcomes resulting from cardiac rehabilitation programs in phase I and II in the last five years. This study is characterized by a literature search, performed through periodic located on databases Lilacs, Scielo e Bireme and data published over the last five years. Eight (08) items involving cardiac rehabilitation phases I and II programs were selected and among the benefits found stand out better score for dyspnea, sensation of effort, tidal volume, vital capacity, pain, strength of the muscles responsible for breathing, heart rate, systolic blood pressure, functional capacity, biochemical profile and hence improves the quality of life of individuals. It can be concluded that cardiac rehabilitation protocols involving the association of conventional physiotherapy with incentive and other therapeutic techniques as EPAP, BiPAP and BS provide a satisfactory and individuals who need immediate results.

Keywords: Cardiac rehabilitation. Physical therapy. Exercise.

\footnotetext{
1 Doutor em Pediatria e Saúde da Criança (PUCRS). Professor do Instituto Cenecista de Ensino Superior de Santo Ângelo (Iesa). moraesvargas@yahoo.com.br

2 Fisioterapeuta (Iesa). regisvieira.fisio@yahoo.com.br

3 Fisioterapeuta (Iesa). renato_balbueno@hotmail.com
} 
No Brasil, bem como em outros países, observou-se nos últimos anos uma importante mudança no perfil da mortalidade da população, caracterizado pelo aumento dos óbitos causados por doenças crônicas não transmissíveis (DCNT). Dentre essas DCNT destacam-se as doenças cardiovasculares (DC) (Ribeiro; Cotta; Ribeiro, 2012).

Segundo a Organização Mundial da Saúde (OMS), as DCs são um grupo de patologias que atingem o coração e os vasos sanguíneos (Organização..., 2014). As DCs apresentam o maior índice de mortalidade e morbidade da população brasileira e mundial. No Brasil são responsáveis por cerca de $30 \%$ de todos os óbitos anuais, com o infarto agudo do miocárdio sendo o principal motivo (Titoto et al., 2005). Essas patologias podem levar à invalidez parcial ou total e consequentemente a graves prejuízos ao indivíduo acometido, sua família e sociedade. Assim, são consideradas um problema preocupante de saúde pública (Scachetti et al., 2011).

Entre os fatores de risco com maior importância para patologias cardíacas destacam-se: hipertensão arterial, idade, obesidade, diabetes mellitus, dislipidemias, tabagismo, alcoolismo, estresse e sedentarismo. A hipertensão arterial constitui um dos principais fatores de risco, pois acarreta alterações em todo sistema cardiovascular podendo originar cardiopatias. A hipertensão arterial atinge cerca de 15\% a $20 \%$ da população adulta, podendo chegar a $50 \%$ em idosos, tendo prevalência maior no sexo feminino (Bernardo et al., 2013; Scachetti et al., 2011).

Em muitas situações os indivíduos acometidos por patologias cardíacas são submetidos a um processo cirúrgico. Isso normalmente ocorre pelo fato de não haver mais recursos eficientes para a regressão das obstruções arteriais. A cirurgia cardíaca possui o objetivo de diminuir complicações no sistema musculoesquelético e cardiovascular, com o propósito de retornar às atividades diárias e melhorar a qualidade de vida destes indivíduos, o que se dá com a realização de programas estruturados de reabilitação cardíaca (Titoto et al., 2005).

A Reabilitação Cardíaca (RC) pode ser definida como uma soma de intervenções que asseguram a melhora das condições físicas, psicológicas e sociais de pacientes com doenças cardiovasculares pós-aguda e crônica. Grande parte do sucesso dos programas de reabilitação cardíaca, contudo, é devida à terapia baseada no exercício físico, esta considerada a estratégia central destes programas (Ricardo; Araújo, 2006).

A RC pode dividir-se em quatro fases. Para se dar início ao processo de tratamento é necessário a liberação do médico assistente e a estabilidade hemodinâmica do paciente. A fase 1 é aplicada ao paciente internado, destinada à recuperação de pacientes hospitalizados por descompensação clínica de natureza cardiovascular, pulmonar e metabólica que envolvem infarto do miocárdio ou cirurgia de revascularização miocárdica, intervenções coronárias percutâneas por técnica de balão ou implante de stent, cirurgias para valvopatia, cirurgias para cardiopatia congênita, transplante cardíaco, angina do peito de caráter estável e paciente com fatores de risco para doença coronária. A fase 2 é a primeira etapa extra-hospitalar, iniciando-se imediatamente após a alta, com duração prevista entre três a seis meses, podendo em algumas situações se estender por mais tempo. Deve ser individualizada e supervisionada por uma equipe multidisciplinar. As fases 3 e 4 destinam-se a atender imediatamente pacientes liberados da fase 2 , sendo caracterizadas como trabalho a longo prazo (duração indeterminada), com realização de atividades para que possam manter e melhorar a aptidão física (Cortez et al., 2006; Nunes, 2010).

A fisioterapia possui um papel fundamental no processo de RC, com ênfase de atuação nas fases $1 \mathrm{e}$ 2. O exercício físico e a biomecânica são fundamentais para eliminar ou reduzir as limitações físicas e sociais advindas das patologias cardíacas (Mair et al., 2008). Na fase 1 o fisioterapeuta deve trabalhar com exercícios físicos de baixa intensidade e também educar o paciente quanto aos fatores de risco associados. Além do fisioterapeuta, a equipe profissional ideal deve ser composta por médico, enfermeiro, nutricionista e psicólogo (Cortez et al., 2006).

No período inicial do pós-operatório ocorre uma significativa redução no desempenho da musculatura respiratória devido à dor gerada pelo processo cirúrgico e aos efeitos dos analgésicos (Ferreira; Ro- 
drigues; Evora, 2009). Tendo em vista o quadro de disfunção pulmonar, a fisioterapia respiratória visa a reverter ou amenizar o quadro álgico, evitando o desenvolvimento de complicações pulmonares. Uma grande variedade de técnicas está à disposição dos profissionais fisioterapeutas (fortalecimento da musculatura respiratória, reexpansão pulmonar, higiene brônquica, etc.) objetivando a reabilitação cardíaca do indivíduo (Renault; Costa-Val; Rossetti, 2008).

Durante a fase 2 as sessões devem ser supervisionadas pelo fisioterapeuta, mas também devem ter o suporte da equipe multidisciplinar. O programa das atividades deve ser individualizado, em termos de intensidade, duração, frequência, modalidade de treinamento e progressão dos exercícios. No decorrer da sessão deve ocorrer o acompanhamento da frequência cardíaca, da pressão arterial, da saturação de oxigênio e da glicemia, além da monitoração eletrocardiográfica (Cortez et al., 2006). Os exercícios aeróbicos que utilizam as extremidades superiores e inferiores, como os realizados no cicloergômetro e na bicicleta ergométrica, são as atividades mais comumente usadas para os pacientes cardíacos com ênfase na função cardiovascular e cardiorrespiratória (Santos et al., 2005).

Uma vez que os cardiopatas apresentam maiores riscos na prática de exercícios físicos quando não controlados por profissionais da área da saúde, entende-se que a RC criteriosa realizada multidisciplinarmente é um processo fundamental para o sucesso do tratamento. Assim sendo, esta revisão bibliográfica visa a identificar os resultados obtidos pelos programas de reabilitação cardíaca em estudos realizados nos últimos cinco anos, a fim de ressaltar a importância da reabilitação cardíaca na qualidade de vida desses pacientes e a atuação essencial do fisioterapeuta.

\section{MATERIAL E MÉTODO}

O presente estudo consiste em uma revisão da literatura, realizada por meio de uma pesquisa nos bancos de dados Lilacs, Scielo e Bireme. Foram selecionados somente artigos publicados a partir do mês de setembro de 2009 até o mês de setembro de 2014, no idioma português, envolvendo ensaios clínicos randomizados de programas de reabilitação cardíaca nas fases 1 e 2, em seres humanos, em que as palavras-chave deviam constar no título ou resumo. As palavras-chave utilizadas foram reabilitação cardíaca, fisioterapia e exercício físico, além de suas combinações. Foram excluídas deste estudo outras revisões bibliográficas, estudos de caso e também aqueles que não se enquadraram nos objetivos desta revisão. Todos os artigos oriundos das buscas realizadas tiveram o seu título e resumo analisados. Em caso de dúvidas sobre a inclusão foi realizada a verificação do texto completo.

A análise dos dados foi feita a partir de uma prévia leitura dos resumos e seleção dos artigos, realizando uma sistematização da análise dos métodos e dos principais resultados dos estudos. Para a categorização dos estudos utilizou-se a técnica de análise de conteúdo, incluindo etapas como: pré-análise, exploração do material e tratamento dos resultados (Bardin; Reto; Pinheiro, 1979). Desta forma, os principais aspectos de cada artigo selecionado foram colocados em uma tabela, para a realização de uma análise crítica dos parâmetros avaliados e dos resultados neles contidos.

\section{RESULTADOS}

Por meio de uma busca nas bases de dados citadas anteriormente foram encontrados 140 artigos, dos quais 26 foram pré-selecionados de acordo com os critérios de inclusão, e, destes, 18 foram excluídos por repetições nas bases de dados, metodologia e ano de publicação. Ao todo 8 artigos foram incluídos nesta revisão, como mostra a Figura 1. 
Figura 1 - Fluxograma dos estudos selecionados sobre Reabilitação Cardíaca

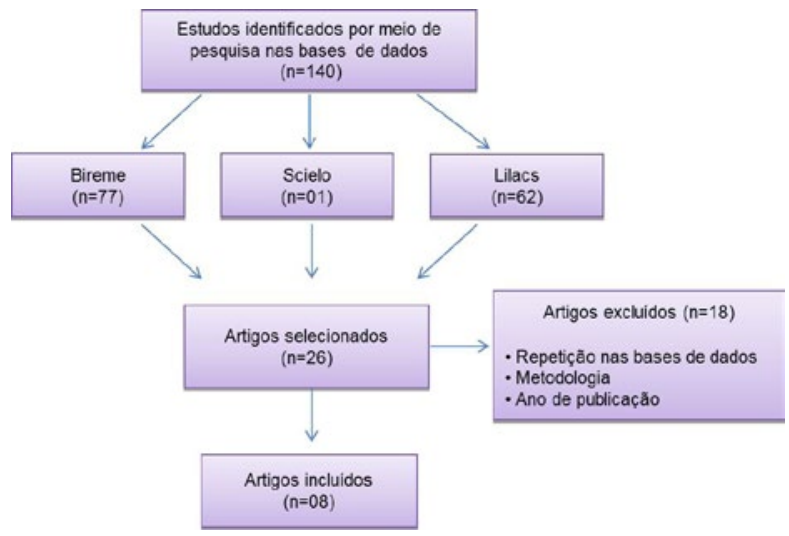

Fonte: Bases de dados Bireme, Scielo e Lilacs

Os protocolos de reabilitação utilizados e os principais resultados descritos nos artigos incluídos podem ser observados na Tabela 1.

Tabela 1 - Estudos com protocolos de Reabilitação Cardíaca fases 1 e 2

\begin{tabular}{|c|c|c|c|}
\hline $\begin{array}{l}\text { AUTOR/ } \\
\text { ANO }\end{array}$ & $\begin{array}{c}\text { MOTIVO } \\
\text { DA INTER- } \\
\text {-VENÇÃO }\end{array}$ & $\begin{array}{c}\text { PROTOCOLO } \\
\text { DE REABILI- } \\
\text { TAÇÃO }\end{array}$ & RESULTADOS \\
\hline $\begin{array}{c}\text { Ferreira et } \\
\text { al., } 2010\end{array}$ & $\begin{array}{l}\text { Revascula- } \\
\text { rização do } \\
\text { Miocárdio }\end{array}$ & $\begin{array}{l}\text { Uso de espirome- } \\
\text { tria de incentivo } \\
\text { (EI) associada } \\
\text { com pressão posi- } \\
\text { tiva expiratória na } \\
\text { via aérea (Epap). }\end{array}$ & $\begin{array}{c}\text { Melhora no } \\
\text { escore para } \\
\text { dispneia, na sen- } \\
\text { sação de esforço } \\
\text { e na qualidade } \\
\text { de vida. }\end{array}$ \\
\hline $\begin{array}{c}\text { Matheus et } \\
\text { al., } 2012\end{array}$ & $\begin{array}{l}\text { Revascula- } \\
\text { rização do } \\
\text { Miocárdio }\end{array}$ & $\begin{array}{l}\text { Fisioterapia con- } \\
\text { vencional e trei- } \\
\text { namento muscular } \\
\text { inspiratório com } \\
\text { threshold IMT. }\end{array}$ & $\begin{array}{c}\text { Recuperação do } \\
\text { volume corrente } \\
\text { e capacidade } \\
\text { vital no } 3 \text { PO. }\end{array}$ \\
\hline $\begin{array}{l}\text { Franco et } \\
\text { al., } 2011\end{array}$ & $\begin{array}{l}\text { Revascula- } \\
\text { rização do } \\
\text { Miocárdio }\end{array}$ & $\begin{array}{l}\text { Aplicação de } \\
\text { BiPAP associada } \\
\text { à fisioterapia } \\
\text { respiratória con- } \\
\text { vencional. }\end{array}$ & $\begin{array}{l}\text { Melhora na ca- } \\
\text { pacidade vital. }\end{array}$ \\
\hline $\begin{array}{c}\text { Dias et al., } \\
2011\end{array}$ & $\begin{array}{l}\text { Revascula- } \\
\text { rização do } \\
\text { Miocárdio }\end{array}$ & $\begin{array}{l}\text { Técnica BS (brea- } \\
\text { th stacking), asso- } \\
\text { ciada à fisioterapia } \\
\text { convencional. }\end{array}$ & $\begin{array}{l}\text { Maior mobiliza- } \\
\text { ção de volume } \\
\text { inspiratório, } \\
\text { com recuperação } \\
\text { total dos valores } \\
\text { pré-operatórios } \\
\text { até o segundo } \\
\text { dia de pós-ope- } \\
\text { ratório. }\end{array}$ \\
\hline $\begin{array}{l}\text { Lima et al., } \\
2011\end{array}$ & $\begin{array}{l}\text { Revascula- } \\
\text { rização do } \\
\text { Miocárdio }\end{array}$ & $\begin{array}{c}\text { Analgésicos, fisio- } \\
\text { terapia do hospital } \\
\text { e Tens. }\end{array}$ & $\begin{array}{l}\text { Controle da dor } \\
\text { pós-operatória } \\
\text { em pacientes no } \\
1 \text { PO e melhora } \\
\text { da força muscu- } \\
\text { lar respiratória. }\end{array}$ \\
\hline
\end{tabular}

\begin{tabular}{|c|c|c|c|}
\hline $\begin{array}{l}\text { Hiss et al., } \\
\quad 2012\end{array}$ & $\begin{array}{l}\text { Infarto Agu- } \\
\text { do do Mio- } \\
\text { cárdio }\end{array}$ & $\begin{array}{l}\text { Exercícios } \\
\text { respiratórios e } \\
\text { exercícios físicos } \\
\text { dinâmicos. }\end{array}$ & $\begin{array}{c}\text { Aumento da FC } \\
\text { e PAS e altera- } \\
\text { ções autonômi- } \\
\text { cas. }\end{array}$ \\
\hline $\begin{array}{l}\text { Berry; } \\
\text { Cunha, } \\
2010\end{array}$ & $\begin{array}{l}\text { Infarto Agu- } \\
\text { do do Mio- } \\
\text { cárdio }\end{array}$ & $\begin{array}{l}\text { Exercícios aeró- } \\
\text { bios em esteira } \\
\text { rolante e bicicleta } \\
\text { ergométrica, } \\
\text { exercícios de } \\
\text { força, exercícios } \\
\text { de flexibilidade } \\
\text { e exercícios de } \\
\text { alongamento. }\end{array}$ & $\begin{array}{l}\text { Melhora na ca- } \\
\text { pacidade funcio- } \\
\text { nal, na eficiência } \\
\text { do sistema } \\
\text { cardiorrespira- } \\
\text { tório e no perfil } \\
\text { bioquímico. }\end{array}$ \\
\hline $\begin{array}{c}\text { Bachur et } \\
\text { al., } 2009\end{array}$ & $\begin{array}{l}\text { Cardiopatas } \\
\text { sem patolo- } \\
\text { gia definida } \\
\text { no estudo }\end{array}$ & $\begin{array}{l}\text { Exercício aeró- } \\
\text { bico na bicicleta } \\
\text { ergométrica e } \\
\text { após treinamento } \\
\text { de força com re- } \\
\text { sistência elástica. }\end{array}$ & $\begin{array}{l}\text { Ocorreu au- } \\
\text { mento na PAD } \\
\text { pós-exercício } \\
\text { de resistência } \\
\text { elástica. }\end{array}$ \\
\hline
\end{tabular}

Legenda: PAD $=$ Pressão Arterial Diastólica; 3 PO = Terceiro dia de Pós-Operatório; 1 PO = Primeiro dia de Pós-Operatório; FC = Frequência Cardíaca; PAS = Pressão Arterial Sistólica.

Fonte: Artigos encontrados nas buscas realizadas nas bases de dados.

\section{DISCUSSÃO}

Entre os artigos selecionados a grande maioria dos estudos que aplicaram programas de reabilitação cardíaca $(55,5 \% \mathrm{n}=05)$, foram atribuídos ao pós-operatório de revascularização do miocárdio (Ferreira et al., 2010; Matheus et al., 2012; Franco et al., 2011; Dias et al., 2011; Lima et al., 2011). Pode-se observar também que $22,2 \%(n=02)$ dos estudos foram abordados no infarto agudo do miocárdio (Hiss et al., 2012; Berry; Cunha, 2010) e outros $11,1 \%(\mathrm{n}=01)$ foram realizados com cardiopatas em geral, sem patologia definida (Bachur et al., 2009).

Dos 5 estudos que abordaram os programas de reabilitação cardíaca no pós-operatório de revascularização do miocárdio, 3 utilizaram espirometria de incentivo (Ferreira et al., 2010; Matheus et al., 2012; Dias et al., 2011).

Ferreira et al. (2010) empregaram o incentivador Voldyne 5000 associado com pressão positiva expiratória na via aérea (Epap) e Matheus et al. (2012) submeteram os indivíduos ao treinamento muscular 
inspiratório com threshold IMT, ambos associados com a fisioterapia convencional (FCo) e em comparação com somente à $\mathrm{FCo}$, evidenciaram que com os incentivadores houve melhora no escore para dispneia e na sensação de esforço, maior recuperação do volume corrente e da capacidade vital. Por fim, Dias et al. (2011) compararam o incentivador respiratório Voldyne 5000 com a técnica BS (breath stacking), a qual consiste em esforços inspiratórios utilizando uma máscara facial acoplada a uma válvula unidirecional, ambos associados com a FCo e em comparação também com somente a FCo relataram que a técnica BS promove maior mobilização de volume inspiratório, com recuperação total dos valores pré-operatórios até o segundo dia de PO.

Dos artigos que não fizeram uso de incentivadores (Franco et al., 2011; Lima et al., 2011), Franco et al., (2011), em seu estudo, os indivíduos com revascularização do miocárdio, foram submetidos a ventilação não invasiva com dois níveis de pressão positiva (Bipap) associada à fisioterapia respiratória convencional (FRC) e em comparação com apenas à FRC, observaram uma melhora na capacidade vital do grupo que utilizou Bipap. Já Lima et al. (2011) utilizaram a estimulação elétrica nervosa transcutânea (Tens) juntamente com terapia analgésica e fisioterapia do hospital e ao confrontarem com apenas a terapia analgésica associada com a fisioterapia do hospital, evidenciaram que a Tens mostrou-se eficaz no controle da dor pós-operatória em pacientes no $1^{\circ} \mathrm{PO}$ de cirurgia de revascularização do miocárdio, evitando o uso excessivo de analgésicos, bem como na melhora da força muscular respiratória, principalmente na pressão expiratória máxima (PEmáx).

Dois estudos abordaram programas de reabilitação cardíaca no infarto agudo do miocárdio (Hiss et al., 2012; Berry; Cunha, 2010). Em seu estudo Hiss et al. (2012) submeteram os indivíduos a um protocolo padrão de reabilitação cardíaca na fase 1 , realizado na posição supina, que incluía dez minutos de repouso pré e pós-exercícios, seguidos de quatro minutos de exercícios respiratórios (padrão diafragmático e respiração em três tempos) e cinco minutos de exercícios físicos dinâmicos (flexo-extensão e abdução-adução dos quadris; flexo-extensão do quadril e dos joelhos associados). Observaram também alterações na modulação autonômica da FC, bem como repercussões hemodinâmicas nesses pacientes, sem, no entanto, ocasionar qualquer intercorrência clínica, ou presença de qualquer sinal e/ ou sintoma de intolerância ao esforço.

Já Berry e Cunha, (2010) utilizaram exercícios aeróbicos em esteira e bicicleta ergométrica, com duração entre 20 e 40 minutos por sessão, exercícios de força, de flexibilidade e de alongamento. Após seis meses de tratamento, os indivíduos obtiveram um aumento da capacidade funcional, aumento do pulso de oxigênio, elevação da Pressão Arterial Sistólica (PAS) não modificando a Pressão Arterial Diastólica (PAD) e melhora na eficiência ventilatória. Houve também melhora no perfil lipídico e glicose (bioquímico).

Por fim, Bachur et al., (2009) aplicaram um programa de reabilitação cardíaca em indivíduos cardiopatas, com patologias não definidas. Todos foram submetidos a exercício aeróbico na bicicleta ergométrica e após 12 sessões com intensidade de $60 \%$ de frequência cardíaca máxima foi dado início ao treinamento de força com resistência elástica, em que o grupo muscular escolhido foi o quadríceps. Após o término do estudo concluíram que comparando o treinamento aeróbico com o de resistência elástica, analisados em repouso, não se observou diferença na FC e na PAS, porém verificou-se aumento na PAD pós exercício com resistência elástica.

Todos os estudos em questão abordaram diferentes protocolos de RC e evidenciaram resultados benéficos aos indivíduos. Dentre os achados positivos nos artigos selecionados destacam-se: relação ao escore para dispneia, sensação de esforço, volume corrente, capacidade vital, dor, força dos músculos responsáveis pela respiração, FC, PAS, capacidade funcional, perfil bioquímico e consequentemente na qualidade de vida dos indivíduos (Ferreira et al., 2010; Matheus et al., 2012; Franco et al., 2011; Dias et al., 2011; Lima et al., 2011; Hiss et al., 2012; Berry; Cunha, 2010). Bachur et al. (2009), porém, encontraram no seu estudo, um aumento na PAD pós-exercício de resistência elástica, resultado este que igualmente contribui para o conhecimen- 
to científico, porém como resultado negativo e um exemplo a não ser seguido pelos profissionais da área.

Esta revisão bibliográfica apresenta algumas limitações metodológicas. Uma delas é o fato abordar de forma geral a atuação da Fisioterapia na reabilitação cardíaca, sem patologia definida previamente. Outra limitação é realização da busca em três bases de dados, utilizando somente o idioma português.

\section{CONCLUSÃO}

Os resultados encontrados nesta revisão confirmam os benefícios dos programas de Reabilitação Cardíaca na abordagem terapêutica de cardiopatas. Os principais achados foram alterações autonômicas e no perfil bioquímico dos indivíduos, melhorando a eficiência do sistema cardiorrespiratório, a capacidade funcional e consequentemente a qualidade de vida. Podemos concluir ainda que a combinação da Fisioterapia convencional associada a incentivadores respiratórios e outras técnicas terapêuticas (Bipap, Epap, técnica breath stacking e Tens) demonstram resultados significativos para a recuperação imediata do indivíduo acometido por alguma afecção cardiovascular. Com isso entende-se que a Fisioterapia tem papel fundamental na Reabilitação Cardíaca.

É muito importante, e necessária, a continuidade de pesquisas nesta área, se possível estudos controlados e randomizados, visando avaliar os efeitos de protocolos de Reabilitação Cardíaca em cardiopatas no intuito de definir protocolos mais adequados, específicos e reutilizáveis em outros estudos.

\section{REFERÊNCIAS}

BACHUR, C. K. et al. Treinamento de resistência elástica em programa de reabilitação cardiovascular. Revista Sociedade de Cardiologia do Estado do Rio de Janeiro (Socerj), v. 22, n. 6, p. 373-378, nov./dez. 2009.
BARDIN, L.; RETO, L. A.; PINHEIRO, A. Análise de conteúdo. Lisboa: Edições 70, 1979.

BERNARDO, A. F. B. et al. Associação entre a atividade física e fatores de risco cardiovasculares em indivíduos de um programa de reabilitação cardíaca. Revista Brasileira de Medicina do Esporte, v. 19, n. 4, p. 231-32, jul./ago. 2013.

BERRY, J. R. S.; CUNHA A. B. Avaliação dos efeitos da reabilitação cardíaca em pacientes pós-infarto do miocárdio. Revista Brasileira Cardiologia, v. 23, n. 2, p. 101-110, mar/abr. 2010.

CERVATO, A. M. et al. Dieta habitual e fatores de risco para doenças cardiovasculares. Revista de Saúde Pública, v. 31, n. 3, p. 227-35, 1997.

CORTEZ, A. A. et al. Diretriz de reabilitação cardiopulmonar e metabólica: aspectos práticos e responsabilidades. Arquivos Brasileiros de Cardiologia, v. 86, n. 1, p. 74-82, jan. 2006.

DIAS, C. M. et al. Três protocolos fisioterapêuticos: efeitos sobre os volumes pulmonares após cirurgia cardíaca. Jornal Brasileiro de Pneumologia, v. 37, n. 1, p. 54-60, 2011.

FERREIRA, G. M. et al. Espirometria de incentivo com pressão positiva expiratória é benéfica após revascularização miocárdio. Arquivos Brasileiros de Cardiologia, v. 94, n. 2, p. 246-251, 2010.

FERREIRA, P. E. G.; RODRIGUES, A. J.; ÉVORA, P. R. B. Efeitos de um programa de reabilitação da musculatura inspiratória no pós-operatório de cirurgia cardíaca. Arquivos Brasileiros de Cardiologia, v. 92, n. 4, p. 275-282, 2009.

FRANCO, A. M. et al. Avaliação da ventilação não-invasiva com dois níveis de pressão positiva nas vias aéreas após cirurgia cardíaca. Revista Brasileira de Cirurgia Cardiovascular, v. 26, n. 4, p. 582-590, 2011.

HISS, M. D. B. S. et al. Segurança da intervenção fisioterápica precoce após o infarto agudo do miocárdio. Revista Fisioterapia em Movimento, v. 25, n. 1, p. 153-63, jan./mar. 2012.

KAISER, S. E. Aspectos epidemiológicos nas doenças coronariana e cerebrovascular. Revista Sociedade de Cardiologia do Estado do Rio de Janeiro (Socerj), v. 17, n. 1, jan./fev./mar. 2004.

LIMA, P. M. B. et al. Estimulação elétrica nervosa transcutânea após cirurgia de revascularização miocárdica. Revista Brasileira de Cirurgia Cardiovascular, v. 26, n. 4, p. 591-596, 2011. 
MAIR, V. et al. Perfil da fisioterapia na reabilitação cardiovascular no Brasil. Fisioterapia e Pesquisa, v. 15 , n. 4 , p. 333-338, out./dez. 2008.

MATHEUS, G. B. et al. Treinamento muscular melhora o volume corrente e a capacidade vital no pós-operatório de revascularização do miocárdio. Revista Brasileira de Cirurgia Cardiovascular, v. 27 n.3, p. 362-369, 2012.

NUNES, R. A. M. Reabilitação cardíaca. São Paulo: Ícone, 2010.

ORGANIZAÇÃO MUNDIAL DA SAÚDE. OMS. Doenças cardiovasculares. Disponível em: <http:// www.who.int/mediacentre/factsheets/fs317/en/>. Acesso em: 3 set. 2014.

RENAULT, J. A.; COSTA-VAL, R.; ROSSETTI, M. B. Fisioterapia respiratória na disfunção pulmonar pós-cirurgia cardíaca. Revista Brasileira de Cirurgia Cardiovascular, v. 2, n. 34, p. 562-569, 2008.

RIBEIRO, A. G.; COTTA, R. M. M.; RIBEIRO, S. M. R. A promoção da saúde e a prevenção integrada dos fatores de risco para doenças cardiovasculares. Revista Ciência e Saúde Coletiva, v. 17, n. 1, p. 7-17, 2012.

RICARDO, D. R.; ARAÚJO, C. G. S. Reabilitação cardíaca com ênfase no exercício: uma revisão sistemática. Revista Brasileira de Medicina do Esporte, v. 12, n. 5, set./out. 2006.

SANTOS, S. D. F. et al. Atividades cardio-respiratórias e publicações em revistas indexadas: avaliação do interesse científico em reabilitação cardíaca. Pulmão RJ, v. 14, n.4, p. 306-309, 2005.

SCACHETTI, F. et al. Incidência e prevalência de hipertensão arterial e diabetes mellitus em profissionais da área da saúde. In: V CONGRESSO MULTIPROFISSIONAL EM SAÚDE, 5., 2011, Londrina. Anais... Londrina: UniFil, jun. 2011.

TITOTO, L. et al. Reabilitação de pacientes submetidos à cirurgia de revascularização do miocárdio: atualização da literatura nacional. Revista Arquivos de Ciências da Saúde, v. 12, n. 4, p. 216-219, out./ dez. 2005.

Recebido em: 5/3/2015

Aceito em: 2/2/2016 